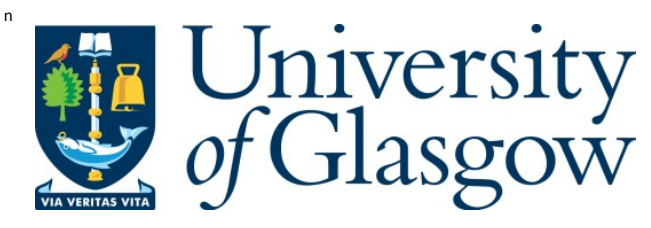

Sengodan, A., Cockshott, W.P., and Cuenca-Garcia, C. (2011) The SIM CA algorithm for processing Ground Penetrating Radar data and its use in locating foundations in demolished buildings. In: RadarCon '11, 23-27 M ay 2011, K ansas City, USA .

Copyright $\odot 2011$ The Higher Education A cademy

A copy can be downloaded for personal non-commercial research or study, without prior permission or charge

Content must not be changed in any way or reproduced in any format or medium without the formal permission of the copyright holder(s)

http://eprints.gla.ac.uk/53542/

Deposited on: 24 N ovember 2014

Enlighten - Research publications by members of the University of Glasgow http://eprints.gla.ac.uk 


\section{The SIMCA algorithm for processing Ground Penetrating Radar data and its use in locating foundations in demolished buildings.}

\author{
Mr. Anand Sengodan and Dr. W. Paul Cockshott \\ Computer Vision and Graphics Group \\ School of Computing Science \\ University of Glasgow \\ Glasgow, G12 8QQ \\ Email: $\{$ sengodan, wpc $\} @$ dcs.gla.ac.uk
}

\author{
Ms. Carmen Cuenca-Garcia \\ Faculty of Arts \\ Department of Archaeology \\ University of Glasgow \\ Glasgow, G12 8QQ \\ Email: c.cuenca-garcia1@ research.gla.ac.uk
}

\begin{abstract}
The main challenge of ground penetrating radar (GPR) based foundation detection is to have an accurate image analysis method. In order to solve the detection problem a system level analysis of the issues involved with the recognition of foundations using image reconstruction is required. The SIMCA ('SIMulated Correlation Algorithm') is a technique based on an area correlation between the trace that would be returned by an ideal point reflector in the soil conditions at the site and the actual trace. During an initialization phase, SIMCA carries out radar simulation using the design parameters of the radar and soil properties. Then SIMCA takes the raw data as the radar is scanned over the ground and in real-time uses a clutter removal technique to remove various clutter such as cross talk, initial ground reflection and antenna ringing. The trace which would be returned by a target under these conditions is then used to form a correlation kernel. The GPR b-scan is then correlated with the kernel using the Pearson correlation coefficient, resulting in a correlated image which is brightest at points most similar to the canonical target. This image is then raised to an odd power $>2$ to enhance the target/background separation. To validate and compare the algorithm, photographs of the building before it was demolished along with processed data using the REFLEXW package were used. The results produced by the $S I M C A$ algorithm were very promising and were able to locate some features that the REFLEXW package were not able to identify.
\end{abstract}

\section{INTRODUCTION}

The west end of Glasgow were the university is located went through a lot of development due to the expansion of the university. A large number of buildings were also demolished and on the area were we conducted the survey, a car park was developed. The authors wanted to test the SIMCA algorithm against data gathered using a GPR and to validate the algorithm using the REFLEXW commercial program.

GPR traces are typically hard to interpret being full of artifacts produced by the imaging modality. GPR integrated with the SIMCA algorithm can provide an image which is easy to interpret. Our algorithm removes these artifacts and allows even unskilled operators to identify the location of buried objects.

The SIMCA algorithm addresses the foundation detection problem by carrying out system level analysis of the issues involved in order to synthesise an image which people can readily understand. SIMCA takes the raw data as the radar is scanned over the ground and in real-time removes various clutter such as cross talk, initial ground reflection and antenna ringing. In order to remove the clutter the mean vector of a number of scans is computed and subtracted from each of the scans and also a windowed average subtraction was used. A clutter removal technique developed by Sengodan and Javadi [2] is used to remove such clutter.

SIMCA compares the trace that would be returned by a ideal point reflector in the soil conditions at the site with the actual traces obtained and from this it works back to the collection of objects that might have generated the observed traces. We obtain the trace that would be generated by the ideal point relector using GprMAX2D v1.5 developed by Antonis [1]; an electronmagnetic simulator for ground probing radar. The simulation solves Maxwells equations using the finite-difference-time-domain method. The simulation takes as input a data file describing soil conditions, domain size, discretization step, time window, details of the buried object, details of radar and the location of the transmitter and receiver. This allowed the derivation of a mathematical model of the response of a point reflector and then to build a deconvolution system.

Once the trace that would be returned by an actual point reflector was worked out from the simulation, an area correlation was performed betweeen the point reflector trace and the actual trace in $M A T L A B^{\circledR}$. This resulted in a correlated image which is brightest at points most similar to the canonical target. Raising the image to an odd power $>2$ enhances the target/background separation.

Various visualization techniques such as false color and mesh generation where used to display the final image by SIMCA.

Figure 1 shows the flowchart of SIMCA algorithm. 


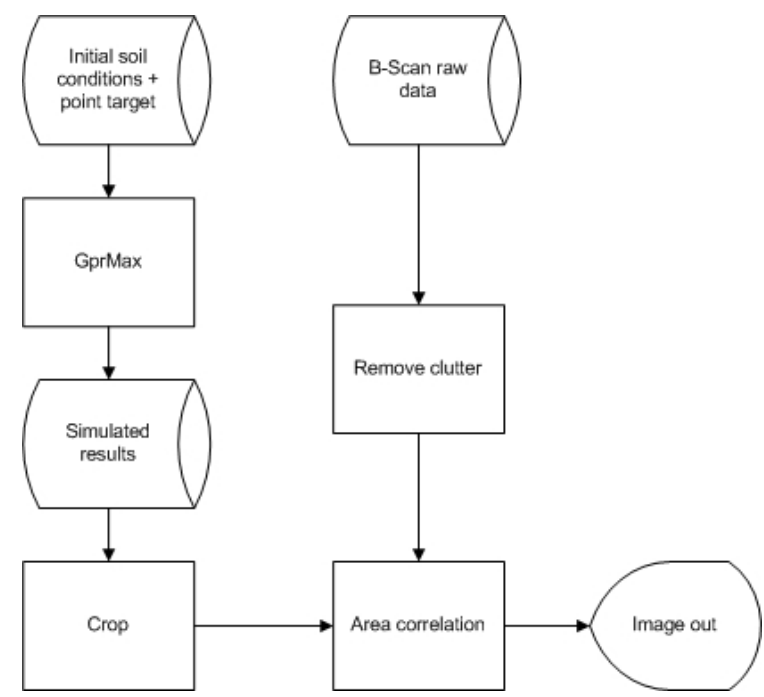

Fig. 1. The flowchart of the SIMCA algorithm

\section{Simulations CARRIED OUT TO DEVElop KeRNELS}

The GPRMAX 2D/3D program, which is a simulation program was run in order to generate a kernel. For running the simulations, a $0.025 \mathrm{~m}$ radius of sphere buried in the same conditions as on the carpark was used because this closely resembled the test conditions and produced a centralised kernel which was large and included a large proportion of the hyperbola.

\section{CORRElation}

Correlation can be used in image-processing to search for specific features or characteristics within an image. The kernel is a two dimensional array but it can be flattened to a vector by row concatenation making it suitable for application of vector correlation. For each pixel position a window centered on the position is selected. The matrix representing the window is flattened to a vector and the correlation vector is computed and then correlated with the kernel.

The advantage of correlation over convolution is that it compensates for differences in gain and black level between the kernel and the area of the image being matched.

We use Pearson's correlation coefficient between two variables which is defined as the covariance of the two variables divided by the product of their standard deviations:

$$
\rho_{X, Y}=\frac{\operatorname{cov}(X, Y)}{\sigma_{X} \sigma_{Y}}
$$

\section{BACKGROUND TO THE SITE}

The car park in Lilybank Gardens is located on a steep hill in the West End of Glasgow (Figure 3). The car park occupies the former site of a row of 19th century terraced houses (Figure 2). During the 20th century the university acquired these properties and in the 1960/70s they were demolished to build the new Business Studies building. The university plans changed and the gap left at the site is currently used as a public car park.

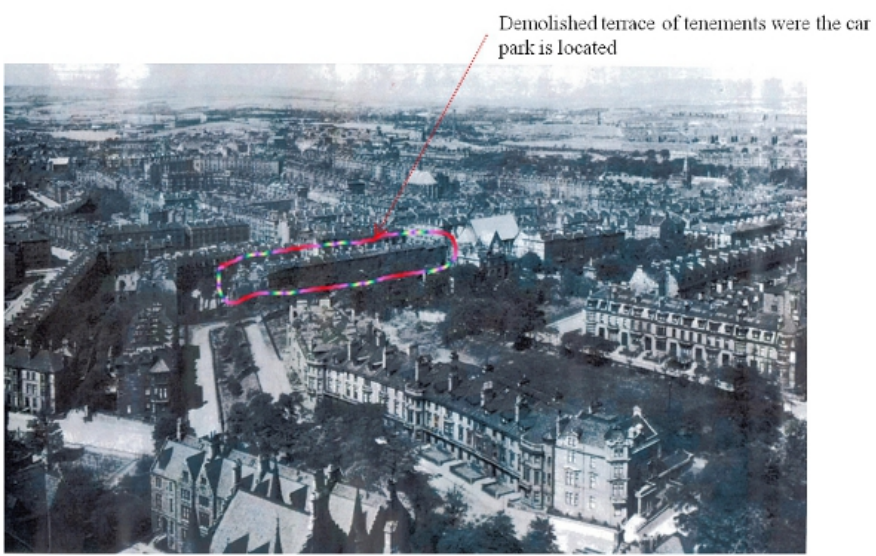

Fig. 2. Photograph showing location of demolished buildings (photograph courteousy of T. R. Annan and Sons)

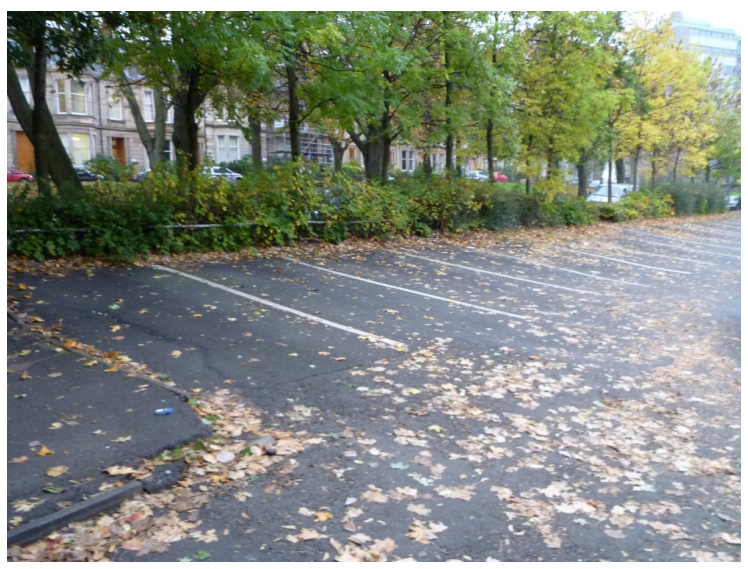

Fig. 3. The existing carpark showing cracks and de-gradation due to existence of foundations

\section{DATA ACQUISITION}

An area of 47.75 square metres was surveyed in the NE of the car park, with the aim of detecting a possible wall foundation suggested by the tarmac surface.

The GPR system used for this investigation was a PulseEKKO 1000 manufactured by Sensors and Software. The system was equipped with a shielded high frequency antenna of $450 \mathrm{MHz}$. Since the tops of structures were expected to be fairly shallow (c.1m or less from the surface), a high frequency antenna was selected in order to balance resolution and depth of penetration requirements.

The survey mode was common offset reflection mode with an antennae separation of $0.25 \mathrm{~m}$. The acquisition was set to continuous mode. A reference tape measure was located along each GPR profile and further parameters were selected in order to ensure correct antennae positioning. Since the target features were expected to be fairly large (wall/foundations) the line spacing used was $0.5 \mathrm{~m}$ with an in line spacing of $0.05 \mathrm{~m}$. This spacing resolution is enough to resolve the requirement of less than a quarter wavelength grid spacing to avoid spatial aliasing and efficient data acquisition for the antenna frequency used. 
The profiles were recorded in parallel and not in zigzag to avoid coupling loss problems as changes in antennae orientation with respect to the ground can potentially cause variation in the data which can be confused with anomalies. The orientation of the GPR profiles was N-S in order to survey in cross-section the target structures. The tarmac surface of the car park was fairly flat, apart from the regular variations due to the underlying structures. This flat surface provided a good contact between the antennae and the ground. Energy loss due to antennae coupling problems was thus avoided. Since the variations on the surface were fairly subtle, no topographic correction was needed.

\section{PROCESSING USING REFLEXW COMMERCIAL PACKAGE}

The GPR data was processed with the REFLEXW package. A fairly standard processing flow was applied to the data and consisted of dewow and background removal, time zero correction and the addition of time based gain.

A three-dimensional block was constructed from the parallel profiles and time-slices extracted from this. Figure 6 shows a time-slice extracted using REFLEXW. These time-slices were also built with the REFLEXW processing package. Figures showing the results obtained using REFLEXW commercial package, both the raw and the processed data are show. Figure 4 shows the unprocessed GPR data and Figure 5 shows the REFLEXW processed data.

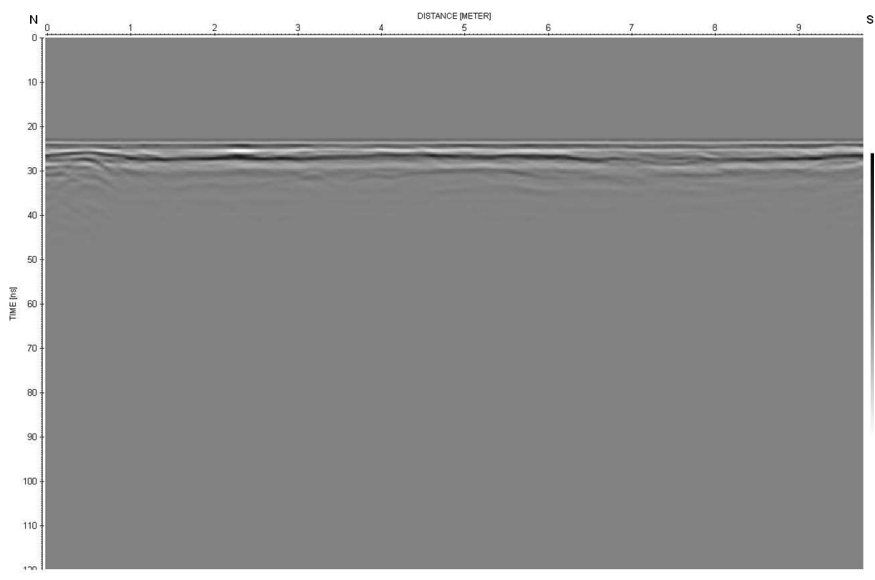

Fig. 4. Picture showing the raw unprocessed GPR data

\section{RESULTS}

Figure 7 shows the results of the SIMCA algoirthm. Figure $7[\mathrm{~A}]$ is the kernel used, whilst figure 7[B] shows the GPR trace after removing the clutter from the raw data. Figure $7[\mathrm{C}]$ shows the result of applying the SIMCA algorithm. Figure 7[D] shows the mesh generated from the correlated results using $M A T L A B$; the peak of the curve as indicated is the location of the foundation. Furthermore figure $7[\mathrm{C}]$ shows the correlated image which is raised to a power of 3. The location of the foundation is as indicated by the boxed area in the diagram.

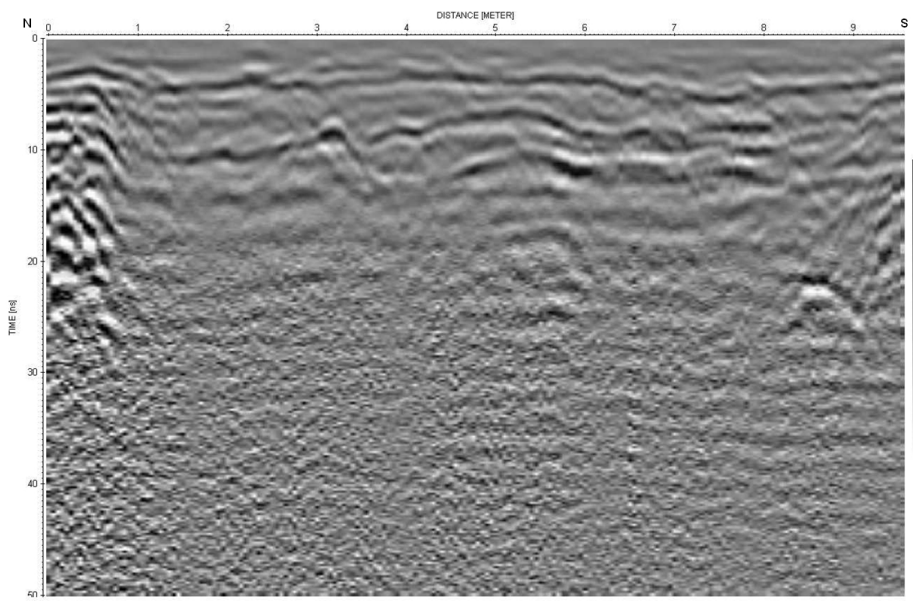

Fig. 5. Picture showing the REFLEXW processed data

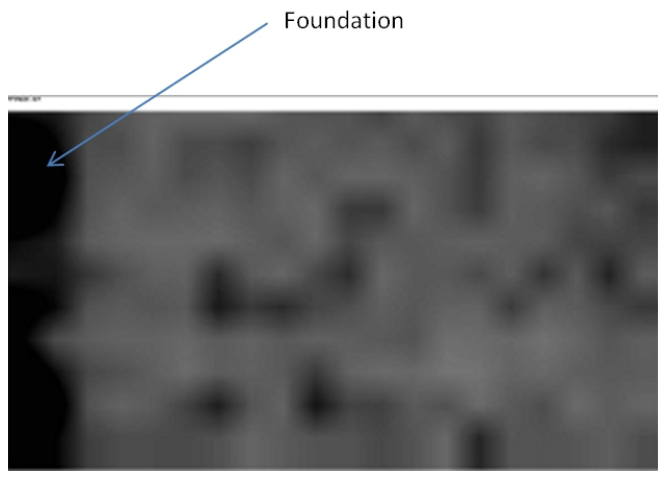

Fig. 6. Picture showing the time slices extracted using the REFLEXW package

The above results were validated using geotechnical diagrams attained by the authors from the archives and they confirmed that the location of the foundations were correct as confirmed by SIMCA. Also using the REFLEXW package the location of the foundation as identified by SIMCA were correct. Figures 5 and 6 confirm the existence of the foundations. Figure 3 shows clearly the cracks that are resultant of the old building foundations which have not been clearly removed. Figure 2 confirms the existence of the terraced building. The car park surface is covered in tarmac and shows regular areas of slight bumps and irregular subsidence of the tarmac. This damage to the surface of the car park appears to be caused by the structural remains of the demolished terraced houses that were present at the site prior the car park construction. Hence, the remains of walls, foundations and other features of the former terraced houses can be subtly perceived on the car park surface.

\section{CONCLUSION}

The proposed SIMCA algorithm is able to locate the location of objects such as building foundations with a high degree of accuracy. The authors have also tested the algorithm on landmine data and the location of landmines were able to be 


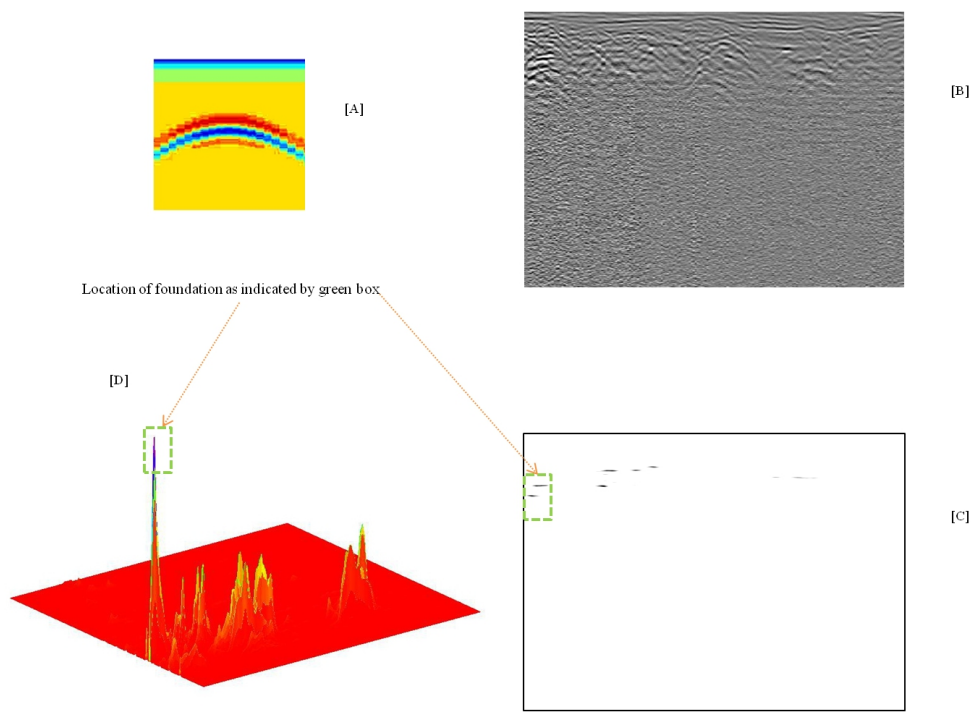

Fig. 7. From clockwise from left- [A]:The picture of the kernel; [B]: Raw cleaned image; [ C]: Correlated image with brightness raised to power of 3; The non-linear operation of raising to the power identifies the correct peak area; [ D]: Mesh generated with MATLAB and the above figure has also been rotated in MATLAB to allow user to predict the location of the target from the visual depth.

located with a high degree of accuracy. The vertical spatial resolution of the technique is better than the horizontal resolution because most of the energy of the kernel section we used is in the vertical direction. The authors anticipate getting even better results using a 3D technique. Also further vizualization and voxel based rendering techniques will improve the clarity of interpretation.

\section{REFERENCES}

[1] Giannopoulos, A., "GprMax software and manual, http://ww.gprmax.org/.

[2] Sengodan, A. and Javadi, A., "Landmine detection using masks on ground penetrating radar images", IRIS 2005.

[3] Daniels, D.J., "Ground Penetrating Radar: 2nd Edition", IEE Radar, Sonar and Navigation Series 151982.

[4] Capineri, L., Grande, P., Temple, J. "Advanced image processing technique for real time interpretation of Ground Penetrating Radar images, L. IEEE Transactions, 1998, vol. 9, pp. 51-59

[5] Daniels David, J., and Allan, R., "Multi-channel landmine detection and radar signal processing using blind de-convolution", Proceedings of the 6th European Radar Conference.

[6] Leonard, L., "Advanced theory of waveguides", Wireless Engineer, 1951.

[7] Dietrich, M., "Theory of dielectric optical waveguides", 2nd edition, c1991 